\title{
Seroprevalence of SARS-CoV-2 IgG antibodies in the staff of the Slovak Academy of Sciences in response to COVID-19 and/or vaccination: situation in August 2021
}

Ivana Kajanova ${ }^{1 \#}$, Lubomira Lukacikova ${ }^{1 \#}$, Lenka Jelenska ${ }^{1}$, Katarina Grossmannova ${ }^{1}$, Zofia Radikova ${ }^{2}$, Miroslav Vlcek ${ }^{2}$, Boris Klempa ${ }^{1}$, Richard Kollar ${ }^{3}$, Katarina Bodova ${ }^{3}$, Juraj Kopacek ${ }^{1}$ Silvia Pastorekova ${ }^{{ }^{*}}$

${ }^{1}$ Biomedical Research Center of the Slovak Academy of Sciences, Institute of Virology, Dubravska cesta 9, 84505 Bratislava, Slovakia; ${ }^{2 B i o m e d i c a l ~ R e s e a r c h ~ C e n t e r ~ o f ~ t h e ~ S l o v a k ~ A c a d e m y ~ o f ~ S c i e n c e s, ~ I n s t i t u t e ~ o f ~ C l i n i c a l ~}$ and Translational Research, 84505 Bratislava, Slovakia; ${ }^{3}$ Comenius University, Faculty of Mathematics, Physics and Informatics, 84248 Bratislava, Slovakia

Received November 11, 2021; accepted November 15, 2021

\begin{abstract}
Summary. - Cross-sectional seroprevalence study of SARS-CoV-2 IgG antibodies was accomplished in the Slovak Academy of Sciences to inform authorities of research institutions about the situation at their workplaces, to assess the risk of next exposure to SARS-CoV-2, and to guide decisions on institutional measures sustaining essential research in evolving epidemic situation. Study participants provided informed consent, anamnestic information, and self-collected dry blood spot samples that were analyzed by ELISA for SARS-CoV-2 S protein-specific IgG antibodies. Relative antibody levels detected in 1928 subjects showed seroprevalence of $84.13 \%$ and led to the following main findings consistent with the current knowledge: (1) mRNA-based vaccines induce better humoral response compared to adenovirus vaccines, (2) antibody levels reflect severity of COVID-19 symptoms, (3) post-COVID vaccination results in marked elevation of IgG levels particularly in asymptomatic and mild cases, (4) antibody levels decrease with increasing time elapsed from vaccination or COVID-19. In addition, data sorting to distinct research institutes and their clustering to three principal scientific sections of the Slovak Academy of Sciences revealed marked differences in seroprevalence, and allowed to identify workplaces with relatively high seropositivity and response rate that can potentially provide a safer working environment than those, where seroprevalence was low or unknown due to low participation. Thus, findings of this study can have direct implications on management decisions during the next pandemic development, with the necessity to keep in mind the phenomenon of time-dependent immunity waning and current spread of more contagious Delta variant of SARS-CoV-2.
\end{abstract}

Keywords: SARS-CoV-2 coronavirus; COVID-19; spike protein; seroprevalence; antibodies; vaccination

\section{Introduction}

Since the first emergence of COVID-19 cases in China in late 2019, SARS-CoV-2 virus has massively spread all

"Corresponding author. E-mail: silvia.pastorekova@savba.sk; phone: +421-2-59302-404. "Equal contribution.

Abbreviations: $\mathrm{Ag}$ test = antigen test; $\mathrm{BMI}=$ body mass index; COVID-19 = Coronavirus disease 2019; $S$ protein = spike protein; SAS = Slovak Academy of Sciences; SARS-CoV-2 = severe acute respiratory syndrome coronavirus 2 over the globe and caused a pandemic of immense dimensions. More than 248 million of confirmed COVID-19 cases and more than 5 million deaths were reported as of November 5, 2021 (WHO COVID Dashboard). Initial nonpharmaceutical interventions were only partially helpful in mitigating the disease transmission. Moreover, SARS-CoV-2 virus multiplication in naïve individuals resulted in development of virus variants of concern with mutations accelerating further spread of infection and/ or weakening virus recognition by humoral immunity (Harvey et al., 2021). 
Unprecedented e ffective development and introduction of vaccines against SARS-CoV-2 virus by the end of 2020 and beginning of 2021 has shown great proficiency in preventing hospitalizations and deaths from severe COVID-19, as documented by both clinical studies and many real-life, nation-wide experiences (Lopez Bernal et al., 2021; Haas et al., 2021; Bar-On et al., 2021).

Both vaccination and COVID-19 have been shown to mount immune responses against the SARS-CoV-2 virus, thereby reducing the pool of individuals susceptible to infection and decreasing the vulnerability of the population to further spreading of virus. Countries with high vaccination rates and/or high prevalence of natural infections can now afford to liberate measures and return to an almost normal lifestyle.

During the first pandemic wave in the spring 2020, Slovakia successfully managed to limit the virus spread in the population via early introduction and good compliance to strict nonpharmaceutical measures. However, the second pandemic wave, rising during October 2020, culminating in January 2021 and then slowly declining by the end of April 2021, had devastating consequences, with an average of 228 COVID-related deaths per 100,000 individuals. That period was characterized by decreasing adherence to measures and strategic decisions, which showed only transient braking effects on the spread of infection. Because of a low proportion of sensitive RT-PCR testing and a high proportion of less sensitive antigen testing, the real epidemiological parameters of the infection remained largely unrecognized and could be judged only according to numbers of hospitalizations and deaths (Kollar and Bodova, 2021).

Starting from December 27, 2020, Slovakia launched vaccination against COVID-19, which showed a promising initial phase, characterized by a low availability of vaccines followed by a decline of interest in vaccination. The decline was associated with a number of governance and communication problems and activities of disinformation scene that resulted in a gradual loss of confidence and an increase of vaccination hesitancy in a relatively high fraction of the population. Interest to get vaccinated almost completely disappeared during the summer period. As no SARS-CoV-2-related serological data from Slovakia is publicly available, a real prevalence of antibodies induced by infection and/or vaccination remains unclear.

To fill the gap, we performed a community-based crosssectional seroprevalence study in employees of the Slovak Academy of Sciences (SAS) in order to help authorities of the SAS research institutions to learn about the situation among the employees at their workplaces, assess the risk of next exposure to SARS-CoV-2 virus, and prepare for the third COVID-19 pandemic wave that was expected to rise in autumn 2021 (what indeed happened).

\section{Subjects and Methods}

Study participants. Study participants were enrolled solely from the employees of the research institutes/centers as well as non-research organizations of the Slovak Academy of Sciences (SAS) exclusively on a voluntary basis. Invitation to participate was distributed via e-mails together with the information for probands, informed consent form, and anamnestic questionnaire. Volunteers expressing their interest to participate in the study received collection set for self-sampling of capillary blood spots with detailed instructions. Collection cards with dryblood samples were returned to the testing laboratory at the Biomedical Research Center of the SAS within 2-3 days following collection and analyzed as described below. The sampling was performed in two phases. The first phase was accomplished within the first two weeks of July 2021 and included fully vaccinated persons (at least 14 days after the second vaccine dose) as well as those who were aware of overcoming COVID-19 (with positive test for SARS-CoV-2). The second phase was performed within the first two weeks of August 2021 and included all other voluntary participants irrespective of whether they were vaccinated, positively tested for SARS-CoV-2 or considered themselves naïve persons with respect to SARS-CoV-2 infection.

Collection of data and ethical approvals. The study was performed under the IMPROVE project (APVV-PP COVID-20-0017) with the ethical approval 07071/2021 provided by an independent Ethics Commission of the Bratislava self-governing region on June 30, 2021. All voluntary participants received written information about the purpose, design, and interpretation of results, and provided signed informed consent as well as anamnestic information including date of birth, gender, height and weight, vaccine type, dates of vaccination and/or date of onset and severity of COVID-19 disease. Severity of the disease was classified as asymptomatic, mild, moderate, severe and critical by probands themselves according to the description of symptoms as defined in the COVID-19 Treatment Guidelines Panel, NIH. All personal data provided by the probands were handled in compliance with the Personal Data Protection Act and other generally binding legal regulations.

Sample collection and preparation for ELISA. Dry blood spot samples were obtained by participants-performed selfcollection of capillary blood by lancet pricking of fingertip using the in-house collection set assembled at the Biomedical Research Center of the SAS. Blood drops were allowed to fall on the collection card and left to dry for 3-4 $\mathrm{h}$ on air. Defined area of the card was punched out and submerged into the sample buffer of the ELISA for $1 \mathrm{~h}$ at $37^{\circ} \mathrm{C}$. The extracted blood sample was then used for the serological analysis using ELISA as described below. The use of dry capillary blood spot samples was validated by parallel testing of venous blood samples of the selected individuals (data not shown).

Serological analysis. Seroprevalence of antibodies to SARSCoV-2 virus was evaluated using an anti-SARS-CoV-2 IgG ELISA 
Table 1. Basic characteristics of the study participants

\begin{tabular}{lcccccc}
\hline Cohort charactertics & Participants & \% & Females (N) & \% & Males (N) & \% \\
\hline Total & 1928 & 100 & 1213 & 62.91 & 715 & 35.08 \\
Vaccinated only & 1300 & 67.43 & 802 & 66.12 & 498 & 69.65 \\
Vaccinated after COVID-19 & 198 & 10.27 & 131 & 10.80 & 67 & 9.37 \\
COVID-19 only & 125 & 6.48 & 76 & 6.27 & 49 & 6.85 \\
No vaccine, no COVID & 299 & 15.51 & 202 & 16.65 & 97 & 13.57 \\
No anamnestic data & 6 & 0.31 & 3 & 0.25 & 3 & 0.42 \\
\hline Age (years), median(IQR) & $48(22)$ & - & $48(20)$ & - & $50(25)$ & - \\
BMI (kg/m), median(IQR) & $24.8(6.37)$ & - & $23.63(6.28)$ & - & $26.21(5.18)$ & - \\
\hline Researchers & 933 & 48.39 & 485 & 39.98 & 448 & 62.66 \\
PhD students & 141 & 7.31 & 95 & 7.83 & 46 & 6.43 \\
Supporting laboratory staff & 167 & 8.66 & 127 & 10.47 & 40 & 5.59 \\
Administrative staff & 228 & 11.83 & 204 & 16.82 & 24 & 3.36 \\
Other operational staff & 459 & 23.81 & 302 & 24.90 & 157 & 21.96 \\
\hline Seropositive & 1622 & 84.13 & 1017 & 83.84 & 605 & 84.62 \\
\hline
\end{tabular}

(EUROIMMUN Medizinische Labordiagnostika AG, Lübeck, Germany), which detects IgG antibodies specifically binding to the SARS-CoV-2-encoded spike protein subunit 1(S1) containing the immunologically relevant receptor binding domain (RBD). The specificity of the anti-SARS-CoV-2 IgG ELISA of $99.6 \%$ and sensitivity in samples collected after day 10 post-symptoms of $94.4 \%$ was determined by the manufacturer. The assay was performed according to the manufacturer's protocol and recommendations, a signal-to-calibrator ratio of $<0.8$ was considered negative $\geq 0.8$ to $<1.1$ borderline, and $\geq 1.1$ positive. Based on the FDA Letter of Authorization including tests acceptable for use in the Manufacture of the High Titer COVID-19 Convalescent plasma, we interpreted the EUROIMMUN test value $\geq 3.5$ as highly positive (De Santis et al., 2021, Hinton, 2021). Participants were informed about their personal test result with notification that it provides only a partial picture of an immune response and cannot determine an individual's risk of subsequent infection.

Statistics. Data were categorized according to selected characteristics and subjected to the statistical analysis. Each analysis included only participants who provided all necessary data. Statistical comparison between groups was first performed using a Kruskal-Wallis test, which detects highly significant differences between vaccine categories ( $p<0.001$ ). To identify the significantly different pairs of categories we performed a multiple comparison test with a Bonferroni correction. The results are shown in the figure insets with different shades of gray encoding individual significance levels (white-non-significant, light gray $-\mathrm{p}<0.05$, medium gray $-\mathrm{p}<0.01$, dark gray - highly significant $\mathrm{p}<0.001)$. Standard least square fitting was used for analysis of temporal dependences of the seropositivity levels. Interquartile range, used in Figs. 1-3, is defined as an interval between the 25 th and the 75 th data percentiles.

\section{Results}

Basic characteristics of the study cohort

A total of 1928 subjects (1213 females and 715 males) with a median age of 48 years and median BMI of $24.8 \mathrm{~kg} / \mathrm{m}^{2}$ participated in the study. The cohort included 933 researchers (48.39\%), 167 supporting laboratory staff members (8.66\%), 228 administrative staff members (11.83\%), 459 other technical and operational staff members (23.81\%), and $141 \mathrm{PhD}$ students (7.31\%). Response rate to the invitation was $49.78 \%$ (52.4\% in the group of employees and $30.45 \%$ in the group of $\mathrm{PhD}$ students). Six participants did not provide any response to anamnestic questionnaire and 54 did not provide information related to BMI. Out of the participants, 1498 persons were vaccinated (77.70\%), and 323 reported COVID-19(16.75\%), with 198 participants belonging concomitantly to both groups. SARS-CoV-2 IgG antibodies were detected in 1622 subjects (84.13\%). There was no significant difference between females and males in age, vaccination status, COVID-19 experience and seropositivity. On the other hand, women showed lower median BMI than men. Basic cohort characteristics are summarized in Table 1.

\section{Vaccination-induced anti-SARS-CoV-2 IgG antibodies}

Out of 1498 vaccinated persons, 802 (53.54\%) received Comirnaty (Pfizer-BioNTech), 215(14.35\%) received Spikevax (Moderna), and 25 persons (1.67\%) claimed mRNA vaccine without specification. Remaining participants received the adenovirus vaccines: Vaxzevria (Oxford- 
Table 2. Composition of the cohort with respect to vaccination status and vaccine type

\begin{tabular}{lcccc}
\hline Vaccine types & Females (N) & Males (N) & Participants (N) & COVID-19 + Vaccine (N) \\
\hline Comirnaty & 521 & 281 & 802 & 115 \\
Spikevax & 136 & 79 & 215 & 27 \\
mRNA (nonspecified) & 17 & 8 & 25 & 5 \\
Vaxzevria & 246 & 181 & 427 & 50 \\
SputnikV & 2 & 7 & 9 & 1 \\
Janssen & 6 & 4 & 10 & 0 \\
Vaxzevria/Comirnaty & 2 & 0 & 2 & 0 \\
CoronaVac & 0 & 2 & 6 & 0 \\
unknown & 3 & 3 & 1498 & 0 \\
\hline All vaccinated & 933 & 565 & & 198 \\
\hline
\end{tabular}
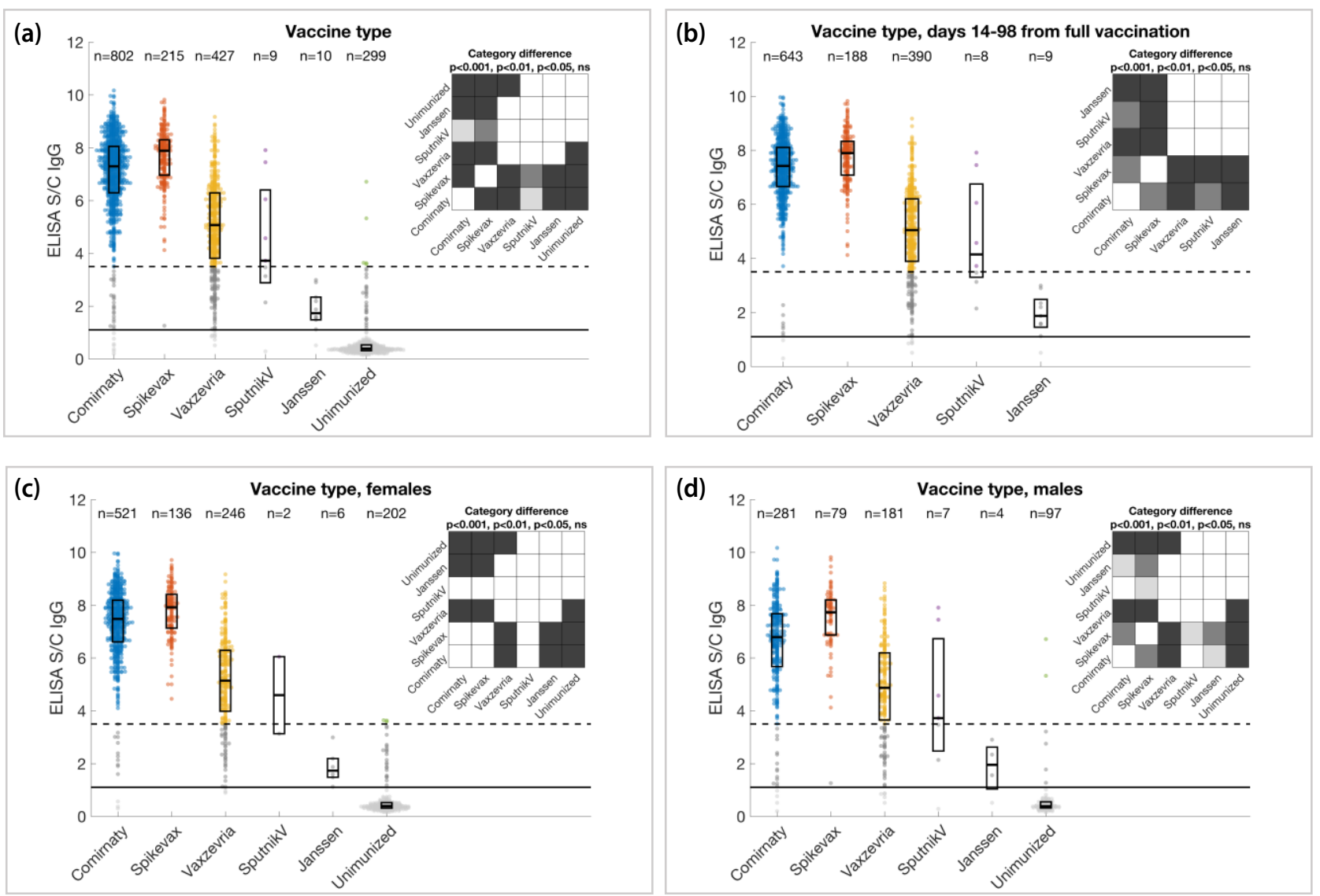

Fig. 1

Relative IgG levels to $S 1$ protein subunit of SARS-CoV-2 in study participants vaccinated by indicated vaccines

(a) data of all vaccinated participants including those with COVID-19, (b) data of all participants who provided samples at 14-98 days from the date of their full vaccination, (c) data of female participants, (d) data of male participants. The data are expressed as a ratio of signal to calibrator (S/C), with the positivity cut-off of 1.1 (full horizontal line) and high positivity cut-off of 3.5 (dashed horizontal line). Median and interquartile range are shown in the boxplots. Statistical comparison between groups was first performed using a Kruskal-Wallis test, which shows highly significant differences between vaccine categories $(\mathrm{p}<0.001)$. To detect the significantly different pairs of categories we performed a multiple comparison test with a Bonferroni correction. The results are shown in the inset with different shades of gray denoting different significance levels (white - non-significant, light gray $-\mathrm{p}<0.05$, medium gray $-\mathrm{p}<0.01$, dark gray - highly significant $-\mathrm{p}<0.001$ ). (a) $[\mathrm{KW}$ test $\mathrm{p}$-value $=1.4130 \mathrm{e}-221],(\mathrm{b})[\mathrm{KW}$ test $\mathrm{p}$-value $=8.7720 \mathrm{e}-109]$, (c) [KW test p-value $=3.4389 \mathrm{e}-147]$, (d) [KW test $\mathrm{p}$-value $=2.0580 \mathrm{e}-71]$. 

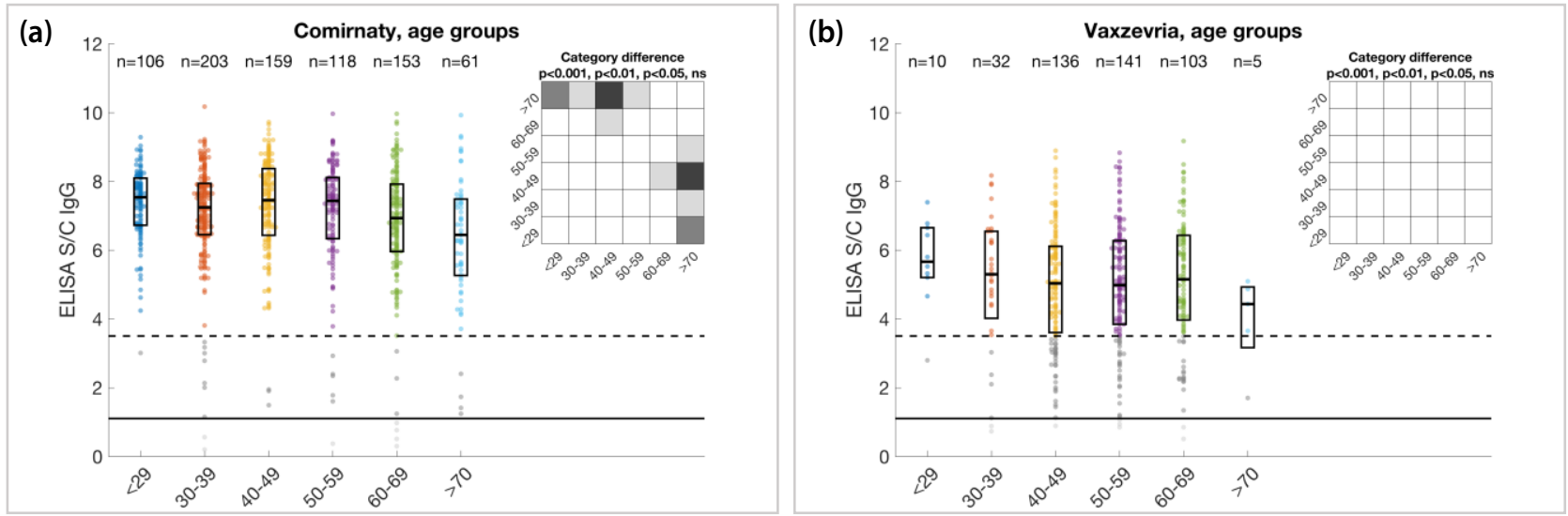

Fig. 2

Relative antibody levels for age categories in vaccinated persons

(a) Participants immunized with Comirnaty vaccine [KW test p-value $=1.5434 \mathrm{e}-04$ ], (b) participants vaccinated with Vaxzevria vaccine $[\mathrm{KW}$ test $\mathrm{p}$-value $=0.4435$ - non-significant] . The data are expressed as a ratio of signal to calibrator $(\mathrm{S} / \mathrm{C})$, with the positivity cut-off of 1.1 (full horizontal line) and high positivity cut-off of 3.5 (dashed horizontal line). Median and interquartile range are shown in the boxplots. Statistical comparison between groups was first performed using a Kruskal-Wallis test, which shows highly significant differences between vaccine categories $(\mathrm{p}<0.001)$. To detect the significantly different pairs of categories we performed a multiple comparison test with a Bonferroni correction. The results are shown in the inset with different shades of gray denoting different significance levels (white - nonsignificant, light gray - $\mathrm{p}<0.05$, medium gray - $\mathrm{p}<0.01$, dark gray - highly significant $-\mathrm{p}<0.001$ ).

AstraZeneca) in 427 cases (28.50\%), SputnikV (Gamaleya Research Institute of Epidemiology and Microbiology) in 9 cases $(0.60 \%)$, and Janssen (Johnson \& Johnson) in 10 cases $(0.67 \%)$. Two participants $(0.13 \%)$ claimed Vaxzevria/ Comirnaty combination and two participants received CoronaVac inactivated virus vaccine by Sinovac Biotech Ltd. Seven participants (0.47\%) indicated the dates of vaccination but have not disclosed the vaccine type (Table 2). At the time of sampling, 79 persons received just the first out of two vaccine shots and were thus only partially vaccinated. Irrespective of the vaccine type, SARS-CoV-2 specific IgG antibodies were detected in $99.2 \%$ vaccinated study participants.

Comparison of the relative levels of S1-protein subunit-specific IgG antibodies induced by full vaccination revealed considerable differences among the vaccine types (Fig. 1a). These differences were apparent also when disregarding the time that elapsed from the second shot (Fig. 1b). The highest relative antibody levels were induced by mRNA vaccines Comirnaty and Spikevax, whereas Vaxzevria and SputnikV induced medium antibody levels. The lowest IgG levels were induced by the one-shot vaccine Janssen. Similar relationships were observed in the subpopulation of females (Fig. 1c) and males (Fig. 1d). Overall, antibody levels in vaccinated persons showed no statistically significant dependence on BMI of the tested subjects (data not shown).

Thus, mRNA vaccines appear to be more effective in inducing antibody response compared to adenovirus vector vaccines. This observation is in line with the published data showing similar vaccine type relationships not only with respect to IgG levels, but also to the levels of neutralization antibodies, which represent only a fraction of the total IgG antibodies specific for S1 protein subunit of SARS-CoV-2 that is considered a surrogate of vaccine protective effect (Mendrone-Junior et al., 2021; Khoury et al., 2021)

Analysis of the relative antibody levels induced by vaccination in relationship to age was performed in the groups of Comirnaty and Vaxzevria recipients that included the largest groups of participants (Fig. 2). The data showed a decrease of IgG levels in the age category of persons older than 70 years. In Comirnaty recipients, the antibody levels in category $>70$ were statistically significantly different from the levels in categories 40-49 and <29 (Fig. 2a). In Vaxzevria recipients, the differences between various age categories were non-significant (Fig. 2b), apparently because the vaccine was not recommended to persons older than 70 years of age and females under 30 years of age at the time of vaccination. The number of samples in these age categories was too small to show any eventual significant statistical difference.

\section{COVID-19-induced anti-SARS-CoV-2 IgG antibodies}

The study cohort included 323 subjects who reported positive results of RT-PCR or Ag test for detection of SARS-CoV-2 virus and overcame COVID-19. Out of these, 

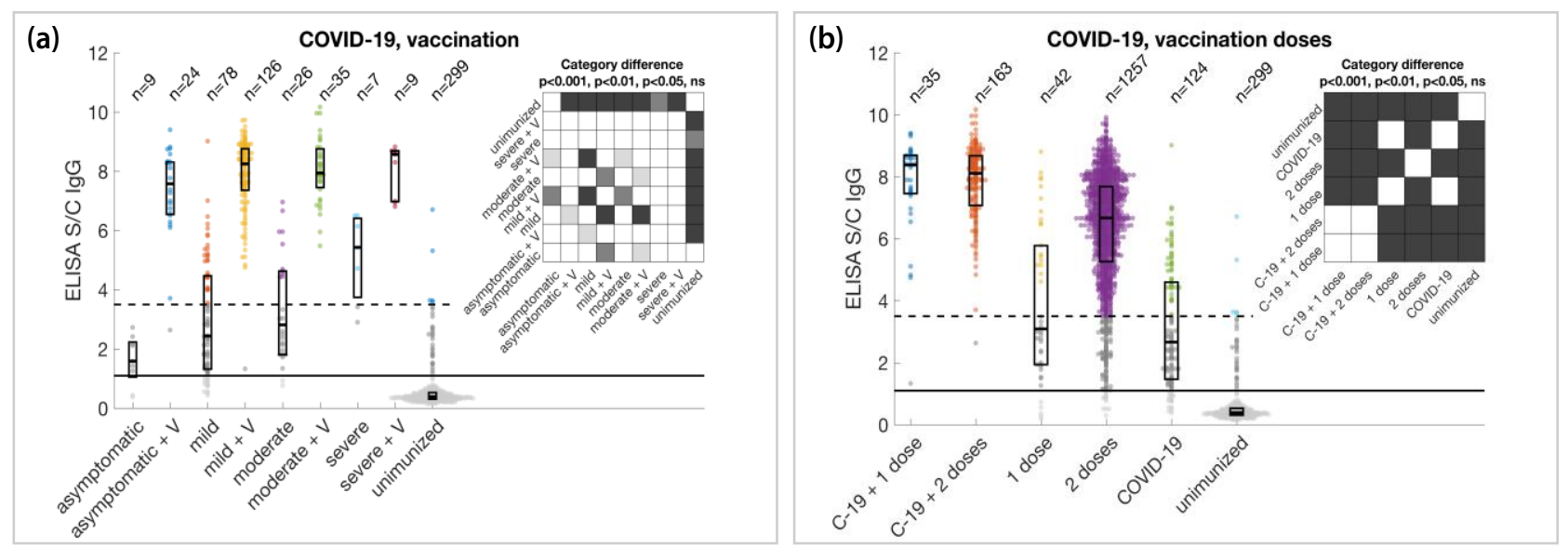

Fig. 3

Relative antibody levels in participants who reported COVID-19 and/or vaccination

(a) Participants stratified according to self-reported severity of COVID-19 symptoms without and with the subsequent vaccination (V, all vaccine types), [KW test $\mathrm{p}$-value $=1.9717 \mathrm{e}-99],(\mathbf{b})$ participants stratified according to number of vaccine doses (irrespective of the vaccine type) without or with the preceding COVID-19 [KW test $\mathrm{p}$-value $=5.9214 \mathrm{e}-212]$. The data are expressed as a ratio of signal to calibrator $(\mathrm{S} / \mathrm{C})$, with the positivity cut-off of 1.1 (full horizontal line) and high positivity cut-off of 3.5 (dashed horizontal line). Median and interquartile range are shown in the boxplots. Statistical comparison between groups was first performed using a Kruskal-Wallis test, which shows highly significant differences between vaccine categories $(\mathrm{p}<0.001)$. To detect the significantly different pairs of categories we performed a multiple comparison test with a Bonferroni correction. The results are shown in the inset with different shades of gray denoting different significance levels (white - non-significant, light gray - $\mathrm{p}<0.05$, medium gray - $\mathrm{p}<0.01$, dark gray - highly significant - $\mathrm{p}<0.001$ ). C-19 in an inlet to (b) corresponds to COVID-19.

198 persons were subsequently vaccinated, whereas 125 did not receive the vaccine. The study participants provided data on the severity of their symptoms based on self-evaluation according to instructions derived from the NIH recommendations (for link see Subjects and Methods). The disease was classified as asymptomatic in case of no manifestation of symptoms, as mild in case of fever, cough, headache and muscle pain, diarrhea, vomiting, loss of smell and/or taste (without shortness of breath); as moderate with increased heart rate more than 90 beats/ min and oxygen saturation more than $93 \%$ in addition to symptoms listed above; as severe with increased heart rate more than 125 beats/min and oxygen saturation less than $93 \%$ in addition to symptoms listed above; and critical with the signs of severe course accompanied by respiratory failure, septic shock, organ failure and hospitalization with intubation or ventilation.

No symptoms were claimed by 33 persons (10.22\%), 204 persons $(63.16 \%)$ described their symptoms as mild, 61 (18.89\%) as moderate, $15(4.64 \%)$ as severe, $3(0.93 \%)$ as critical and 7 persons did not provide the information. As expected, data analysis revealed that antibody levels principally reflected the disease severity in the subgroup of participants who reported COVID-19 and remained without subsequent vaccination. Importantly, in persons who underwent full vaccination by any vaccine following COVID-19, the antibody levels significantly increased ir- respective of the symptoms. This was particularly striking in the category of participants with no, mild and moderate symptoms, who, if not vaccinated, did not develop robust humoral response to natural infection (Fig. 3a). This finding suggested that in those cases, vaccination is very beneficial (or critical) for providing better immune protection from reinfection.

Further comparison of the overall antibody levels assessed in persons who were vaccinated (with any of two-shot vaccines) without overcoming COVID-19, with those who overcame COVID-19 (irrespective of the disease severity) without subsequent vaccination, and those who received one or two doses of any vaccine following the disease with any symptoms showed that one dose of postCOVID-19 vaccination was sufficient to reach the antibody levels equivalent to levels in fully vaccinated persons (Fig. 3b, Levi et al., 2021; Ebinger et al., 2021; Wei et al., 2021). However, so far it is not known whether and how the second post-COVID dose of vaccine affects the longevity of immune protection compared to the first dose. Therefore, this issue requires further investigation.

Relationship between anti-SARS-CoV-2 IgG levels and time elapsed from vaccination or COVID-19

Data from a number of seroprevalence studies in convalescent and/or vaccinated persons demonstrate that 


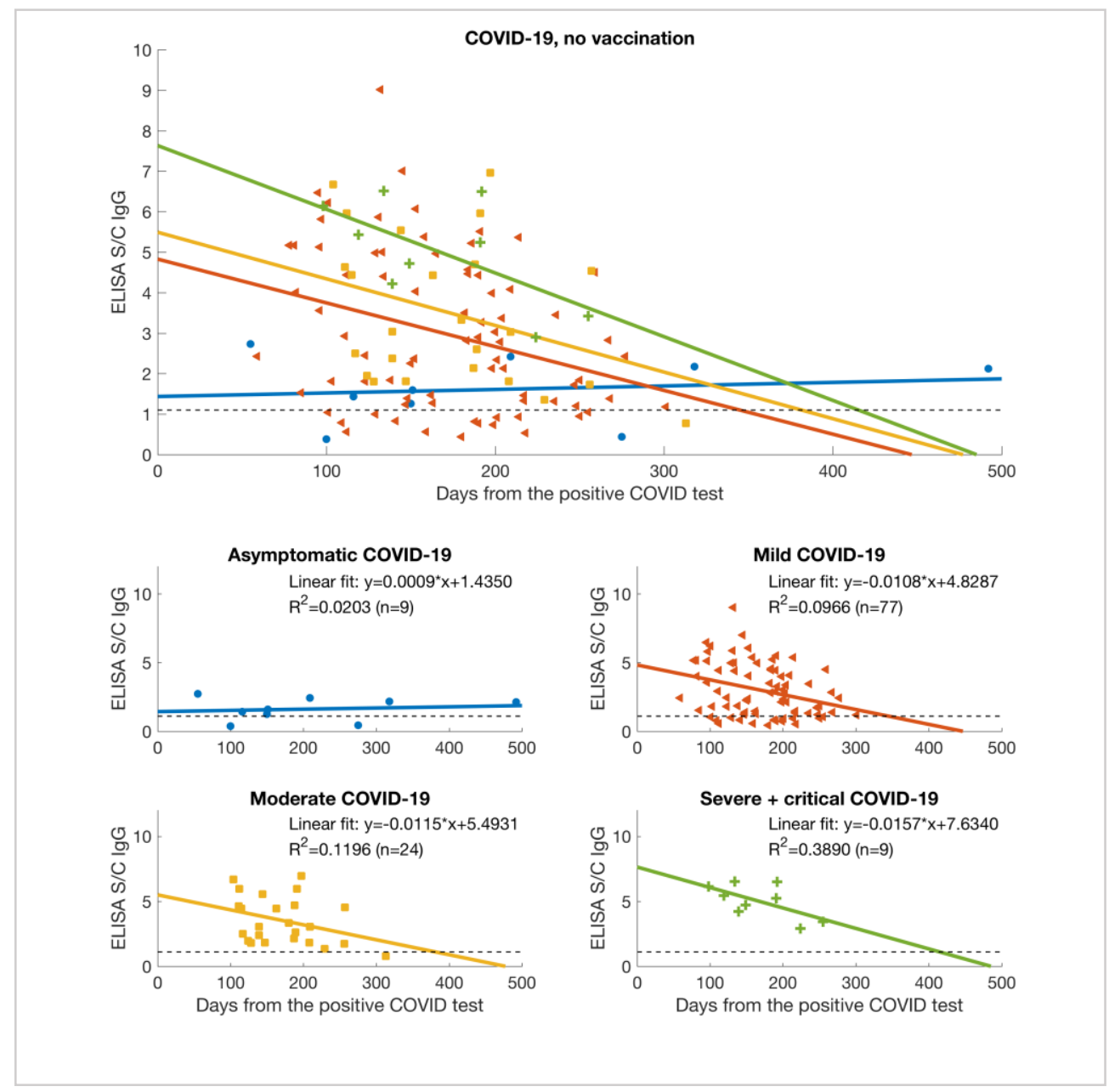

Fig. 4

Relative antibody levels detected in serum samples of participants with COVID-19 versus time elapsed from their positive RT-PCR or Ag test

(Top) All data of study participants who overcome COVID-19. Data for participants were stratified according to self-reported severity of COVID-19 symptoms with the linear regression fits to the data: asymptomatic [regression $p$-value $p=0.715]$, mild [p $=0.00595]$, moderate $[p=0.0979]$, and severe + critical $[p=0.0727]$. Linear regression fit parameters and R-squared are displayed in each figure. The data are expressed as ratio of signal to calibrator (S/C), with the positivity cut-off of 1.1 (dashed horizontal line is used for better visualization).

the SARS-CoV-2 virus-specific IgG antibody levels decline within a period of 6 to 9 months following an induction of the humoral immune response (Alfego et al., 2021; Bayart et al., 2021; Levin et al., 2021). Therefore, the values of relative antibody levels assessed in this study were ordered according to the number of days that elapsed from the onset of clinical symptoms in COVID-19 cases and from the second dose of vaccine, respectively (Fig. 3). It is important to note that each participant provided only a single blood sample collected at different time point from the vaccination and/or detection of SARS-CoV-2 positivity. Therefore, the results do not represent a longitudinal approach, but rather reflect general waning of the humoral arm of immunity with its inherent interindividual variability in the studied cohort.

The subgroup of non-vaccinated persons who overcame COVID-19 entered the study from 70 to 294 days after the detection of infection. The levels of virus-specific IgG antibodies showed a decreasing trend of dependence on the time elapsed from the date of detection of the infection. The estimated average rates of decline were comparable for groups of participants who overcame COVID-19 with mild and moderate symptoms and slightly higher for those with severe and critical symptoms. However, the estimated average initial virus-specific IgG antibody levels induced by COVID-19 varied with severity of the 


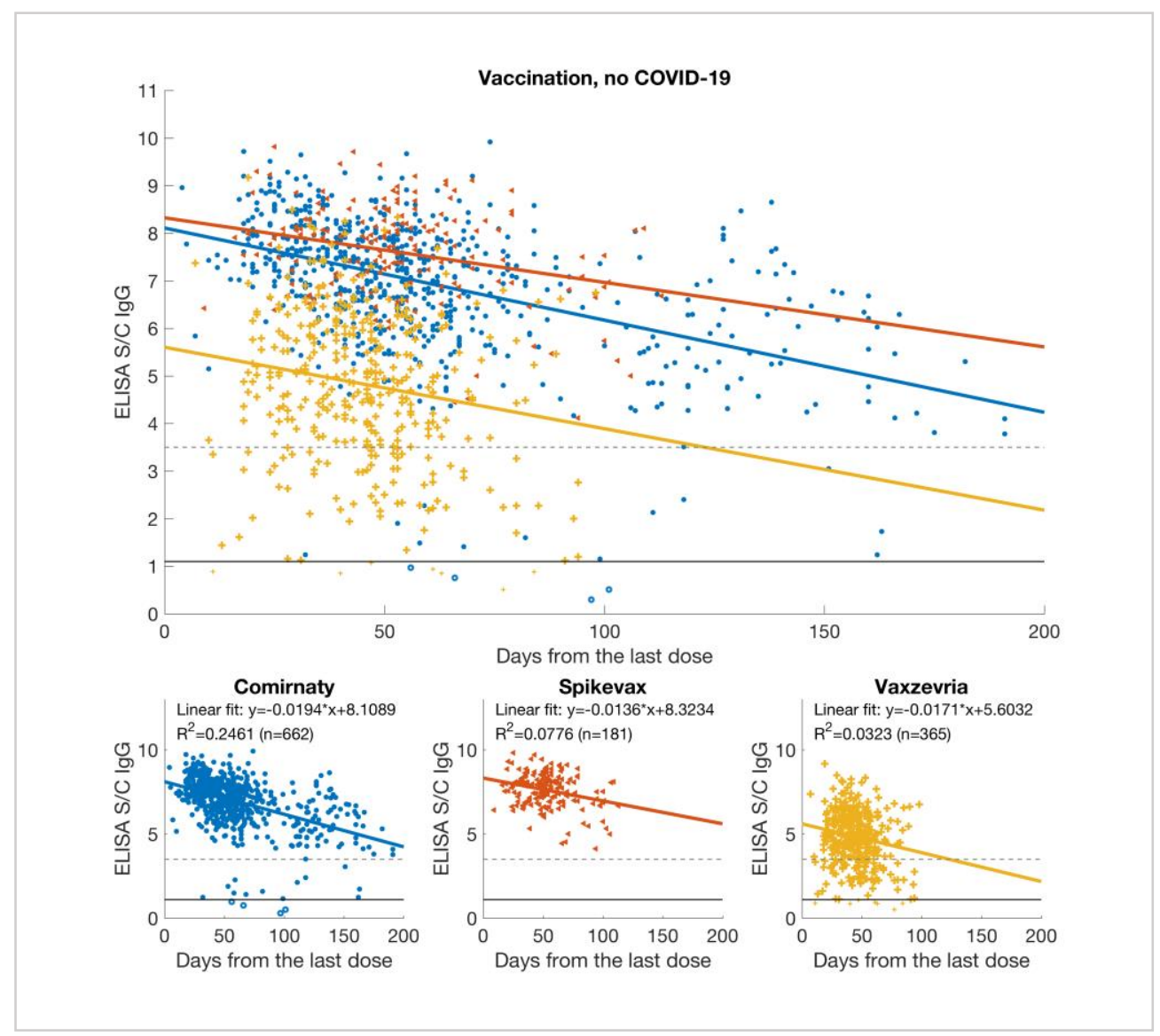

Fig. 5

Relative antibody levels detected in serum samples of vaccinated participants versus time elapsed from the day of their full vaccination

(Top) All data of study participants who were fully vaccinated by Comirnaty, Spikevax and Vaxzevria. Data for participants were stratified according to the type of vaccine with the linear regression fits to the data: Comirnaty [regression $\mathrm{p}$-value $\mathrm{p}=3.54 \mathrm{e}-42$ ], $\mathrm{Spikevax}$ [p = 0.000147], Vaxzevria [ $p=0.00063]$. Linear regression fit parameters and R-squared are displayed in each figure. The data are expressed as ratio of signal to calibrator $(\mathrm{S} / \mathrm{C})$, with the positivity cut-off of 1.1 (dashed horizontal line).

symptoms with the highest value for those with severe and critical symptoms, lower for those with moderate symptoms and the lowest for those with mild symptoms. The small group $(n=9)$ of study participants with asymptomatic COVID-19 showed systematically low levels of antibodies with no significant decreasing temporal trend (Fig. 4).

Similarly, the levels of virus-specific IgG antibodies showed a decreasing trend of dependence on the time elapsed from the date of full vaccination for study participants who did not self-report symptomatic or asymptomatic COVID-19. The estimated rates of decline for subjects vaccinated by individual vaccines (Comirnaty, Spikevax and Vaxzevria) were mutually comparable and slightly higher than those for participants who overcame symptomatic COVID-19. The estimated average initial virus-specific IgG antibody levels induced by full vaccina- tion were similar for the mRNA vaccines and higher than those for groups of participants who overcame COVID-19, including a group with severe and critical COVID-19. Lower initial levels were observed in the group of participants vaccinated by Vaxzevria; comparable initial levels were observed in the group of participants who overcame COVID-19 with moderate symptoms (Fig. 5). The results for fully vaccinated individuals without self-reported COVID-19 confirmed the qualitative results of the analysis presented in Fig. 1, which included all vaccinated study participants regardless of their COVID-19 status.

Comparison of the decreasing trends in the dependence of the virus-specific IgG antibody levels on the time elapsed from full vaccination (reported or projected) in groups of participants with COVID-19 and one or two doses of vaccine are displayed in Fig. 6. Both groups showed identical decline rates comparable with the 


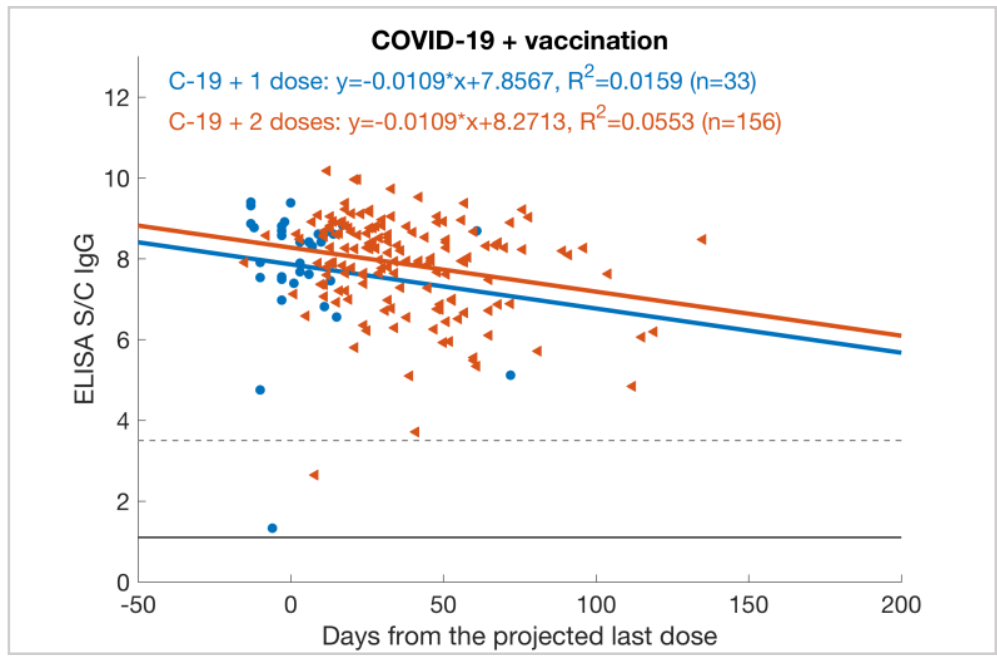

Fig. 6

Relative antibody levels detected in serum samples of participants with COVID-19 who were also vaccinated at least by one dose of a vaccine versus time elapsed from the day of their full vaccination (reported or projected)

Colors (blue and red) indicate participants who received one and two vaccination doses, respectively. [Regression $p$-values $p=0.485$ and $p=0.00312$, respectively]. For study participants with only one dose of vaccine the projected day of full vaccination was set to 28 days after the first dose (Comirnaty and Spikevax) and to 70 days after the first dose (Vaxzevria). Linear regression fit parameters and R-squared are displayed in each figure. The data are expressed as ratio of signal to calibrator (S/C), with the positivity cut-off of 1.1 (dashed horizontal line) and high positivity cut-off of 3.5 (dashed horizontal line).

group of unvaccinated individuals with mild COVID-19 (lower than those fully vaccinated). The estimated initial virus-specific IgG antibody levels induced by a combination of COVID-19 and one or two doses vaccination were comparable with those estimated for the group of fully vaccinated by mRNA vaccines (Comirnaty and Spikevax). A single dose of vaccine in a combination with COVID-19 thus induced similar humoral response. Note that some of the linear fits discussed here were statistically insignificant on the level $5 \%$ (the individual p-values are indicated in figure captions).

\section{Research areas, seroprevalence and vaccination status}

Slovak Academy of Sciences (SAS) comprises 45 research organizations divided into three Scientific Sections according to the research areas: 1 . Physical, Space, Earth and Engineering Sciences (14 research institutes and one research center), 2. Life, Chemical, Medical and Environmental Sciences ( 9 research institutes and 4 research centers), and 3. Social Sciences, Humanities, Arts and Culture (16 institutes and 1 center). In addition, SAS includes non-research bodies, namely the Office of the SAS as the main administration headquarters providing support to the Presidium of the SAS, Central Library and Centre of Operation https://www.sav.sk/?lang=en \& doc=sas-org.

Out of 45 research institutions/centers, 39 participated in the study, corresponding to total 1638 participating employees (response rate 54.20\%). The average seroprevalence of this subpopulation of the study cohort was $85.77 \%$ and was similar among the Scientific Sections, with the highest seropositivity observed among the employees of the life sciences section (Fig. 7). On the other hand, there were significant differences in the response rates, with the highest participation from the Section 2 of Life, Chemical, Medical and Environmental Sciences (64.84\%), followed by the Section 1 of Physical, Space, Earth and Engineering Sciences (47.93\%) and then the Section 3 of Social Sciences, Humanities, Arts and Culture (40.13\%). Obviously, response rates could be also influenced by diverse work schedules of research institutes/staff during summer period when the study was performed. Vaccination status showed similar association: 767 (85.22\%) vaccinated participants from Section 2,403 (80.76\%) from Section 1, and 186 (77.82\%) from Section 3. This may be connected with the awareness of the biological/medical aspects of the pandemic and also by the attributes of research, which mostly depend on a laboratory teamwork in experimentally-oriented research, while it is more individualistic in humanities and social sciences. Due to these characteristics, researchers in natural sciences appear to have inherently higher motivation to accept vaccination as well as nonpharmaceutical protective measures and participate in research studies. Of course, the differences were visible also among the workplaces within the same scientific sections, with several institutes/centers show- 


\begin{tabular}{|ccc|}
\hline \multicolumn{3}{|c|}{$\begin{array}{c}\text { Physical, Space, Earth, and } \\
\text { Engineering Sciences }\end{array}$} \\
$\mathrm{N}$ & $\%$ IgG & $\%$ \\
& positivity & participation \\
24 & 95.83 & 24.49 \\
16 & 93.75 & 36.36 \\
50 & 92.00 & 45.87 \\
34 & 91.18 & 65.38 \\
42 & 90.48 & 84.00 \\
54 & 88.89 & 58.70 \\
30 & 86.67 & 45.45 \\
14 & 85.71 & 35.90 \\
26 & 84.62 & 61.90 \\
39 & 82.05 & 50.65 \\
44 & 79.55 & 37.93 \\
59 & 77.97 & 47.97 \\
35 & 74.29 & 56.45 \\
32 & 56.25 & 45.07 \\
& TOTAL & \\
499 & 83.77 & 47.93 \\
\hline
\end{tabular}

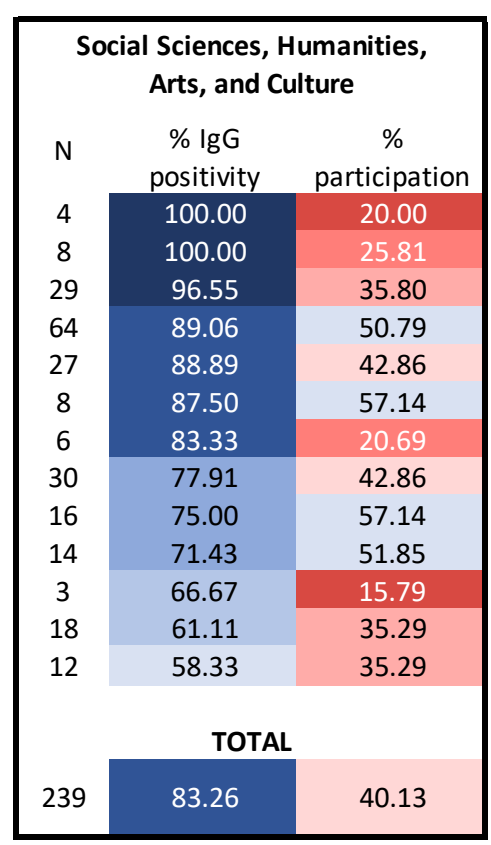

Fig. 7

Seroprevalence and response rate of study participants in the research institutions of the Slovak Academy of Sciences Seroprevalence (expressed as \% of IgG positivity) and response rate (expressed as \% of participation) observed in the individual participating research institutions/centers clustered according to their respective Scientific Sections. Reported vaccination status of the study participants was 403 (80.76\%) in the research organizations of the Scientific Section 1 (left panel), 767 (85.22\%) in Section 2 (central panel) and $186(77.82 \%)$ in Section 3. Color scale corresponds to ten per cent intervals, with gradually fading red color shades for 0-50\% and gradually increasing intensity of blue color shades for 50-100\%. Left columns: $\mathrm{N}=$ number of participants of the individual workplaces (or sections, bottom row). Data demonstrate the highest seroprevalence and response rate among the institutes/centers of the life sciences section.

ing relatively high values in both seroprevalence $(>80 \%)$ and participation (>60\%). These workplaces can provide a safer working environment than those, where seroprevalence was low or unknown due to low participation. Thus, these data can have direct implications on management decisions during the evolving pandemic, with the necessity to keep in mind the phenomenon of time-dependent immunity waning (Bayart et al., 2021; Levin et al., 2021).

\section{Discussion}

This cross-sectional seroepidemiological study was designed to gain insight into a prevalence of IgG antibodies specific for $S$ protein of the SARS-CoV-2 virus in the Slovak Academy of Sciences. SAS is a research institution that provides institutional working environment to researchers as well as supporting technical and administrative staff mostly performing a teamwork (especially in experimental research topics) with active contacts within research groups and supporting units. The study was conducted in the period between the second COVID-19 pandemic wave in Slovakia that ended in May 2021 and the third wave that started in September and is still on the rise in the mid November 2021. The purpose was to obtain information that would help managements of the SAS organizations to deal with the risk of next COVID-19 exposure during autumn/winter, guide planning of specific institutional measures and maintain essential research active in spite of worsening epidemiological situation. However, in considering the results of this study in term of possible future exposures to SARS-CoV-2 infection, it should be taken into account that the current third epidemic wave is caused by more contagious Delta virus variant. This variant is characterized by faster spread and higher infectious loads compared to previous Alpha strain spreading in our population during the second wave. Exposure to Delta creates conditions for higher risk of infection that can be further increased due to waning immunity in those persons who experienced COVID-19 and/or vaccination more than 6-9 months ago (Elliot et al., 2021).

It is important to note that the antibody detection test used in all analyses within this work has limitations. Its semi-quantitative results are nonlinearly skewed both at the high and low ends of its measurement scale range, as it was confirmed by our end-point titration experiments (manuscript in preparation). Nevertheless, a direct fitting 
of the test results is a standard method in the scientific literature. This limitation may influence the overall character and parameters of the fits presented in Figs. 4-6 but does not have an impact on the statistical group comparison results presented in Figs. 1-3 (as they rely on data rank comparison tests). Thus, the identified decreasing trends in the dependence of the antibody levels on the time elapsed from vaccination and/or COVID-19 detection need to be taken with a caution. For the purpose of this crosssectional study and the group comparisons presented here we do not consider this limitation to be significant, although more attention needs to be taken for any similar longitudinal studies. Also some of the linear fits discussed in Figures 4 and 6 are statistically insignificant on the level $5 \%$ ( $p$-value $\mathrm{p}<0.05$ ). This factor may also be influenced by the test limitation, as it contributes to a violation of the assumption of the normally distributed error.

Nevertheless, the present study brought several important findings. From a practical point of view it demonstrated that collection of dry blood spots is a highly effective and reliable sampling approach that allows to obtain, easily store and analyze large numbers of samples during a relatively short period of time. This might be of particular importance when urgent reaction to the ongoing epidemic situation is needed. The study also showed, in accord with other investigations, that both vaccination and natural infection resulting in symptomatic COVID-19 induce detectable humoral immune responses that range from weak to very strong (Racine-Brzostek et al., 2021; Bert et al., 2021). In few subjects, observed weak or even absent humoral response to symptomatic COVID-19 (14.6\%) or vaccination $(0.8 \%)$ can be associated not only with the prolonged period from the moment of immunization, but also with genetic predispositions (agammaglobulinemia or hypogammaglobulinemia), other immunocompromising conditions, comorbidities leading to protein loss, and/or therapeutic interventions leading to immune suppression, e.g. anti-cancer drugs, corticosteroids, antiepileptic drugs, transplantation-related medications etc. (Patel et al., 2019). These health-related conditions can have prolonged negative effect on production of antibodies in general and therefore deserves special attention. In immune-competent subjects, broad interindividual variations in relative IgG antibody levels result from variable settings and dispositions of the immune system (both in case of vaccinated and infected individuals) as well as from a broad scale of symptoms with varying severity (in case of infected persons).

In spite of the variable individual fitness of the immune system, data obtained in this study provided consistent pictures with respect to: (1) ability of mRNA vaccine types to induce better humoral response than the adenovirus vaccines, (2) antibody levels reflecting severity of COVID-19 symptoms, (3) beneficial effect of post-COVID vaccination leading to marked elevation of IgG levels particularly in asymptomatic and mild cases, (4) decreasing antibody levels with increasing time elapsed from vaccination or COVID-19. This consistency does not apply only to the content of this study, but also points to a compliance with other published seroprevalence studies. However, the present findings offer only a partial insight into the adaptive immunity to natural SARS-CoV-2 infection and/or vaccination. It is well established that $\mathrm{T}$ cell-mediated immunity plays a key role in antiviral defense and that individuals with low antibody levels can still develop competent effector and memory T cells capable of protective responses to subsequent SARS-CoV-2 infection (Sette and Crotty, 2021).

Finally, this study uncovered a possible relationship between higher seroprevalence/vaccination status and engagement in research in natural sciences, suggesting that deeper understanding of the causes and consequences of pandemics can facilitate individual decisionmaking. Albeit this assumption cannot be generalized, it underlines an importance of edification of lay public and dissemination of scientific knowledge-based information to build increasing awareness of pandemic threads and opportunities of interventions.

Acknowledgment. This work was funded by the Slovak Research and Development Agency (IMPROVE project, APVVPP-COVID-20-0017), by the Presidium of the Slovak Academy of Sciences, by the Scientific Grant Agency of the Slovak Republic (VEGA 1/0755/19 and 1/0521/20), and by the European Regional Development Fund(OPVaI-VA/DP/2018/1.2.1-08, ITMS 313011V344). The authors wish to thank all colleagues from the Slovak Academy of Sciences for their voluntary participation in the study, to VEDA Publishing house of the SAS for printing the sample collection cards, to Dr. Maria Bartosova and Dr. Ingeborg Rezuchova for their assistance in data registration, and to Ms. Zuzana Lackovicova for the assistance in preparation and distribution of the sample collection sets.

\section{References}

Alfego D, Sullivan A, Poirier B, Williams J, Adcock D, Letovsky $S$ (2021): A population-based analysis of the longevity of SARS-CoV-2 antibody seropositivity in the United States. EClinicalMedicine 36: 100902. Epub 2021 May 24. https://doi.org/10.1016/j.eclinm.2021.100902

Bar-On YM, Goldberg Y, Mandel M, Bodenheimer O, Freedman L, Kalkstein N, Mizrahi B, Alroy-Preis S, Ash N, Milo R, Huppert A (2021): Protection of BNT162b2 Vaccine Booster against Covid-19 in Israel. N. Engl. J. Med. 385, 1393-1400. Epub 2021 September 15. https://doi. org/10.1056/NEJMoa2114255 
Bayart JL, Douxfils J, Gillot C, David C, Mullier F, Elsen M, Eucher C, Van Eeckhoudt S, Roy T, Gerin V, Wieers G, Laurent C, Closset M, Dogné JM, Favresse J (2021): Waning of IgG, Total and Neutralizing Antibodies 6 Months Post-Vaccination with BNT162b2 in Healthcare Workers. Vaccines (Basel) 9, 1092. https://doi.org/10.3390/ vaccines 9101092

Bert NL, Chia WN, Wan WY, Teo AKJ, Chong SZ, Tan N, Tan DSC, Chia A, Tan IB, Kunasegaran K, Chua QX, Abdad MY, Hua Ng AS, Vasoo S, Ang JX, Lee MS, Sun L, Fang J, Zhu F, Cook AR, Aw TC, Huang J, Tam C, Lee FS, Clapham H, Goh EJ, Peou MSS, Tan SP, Ong SK, Wang LF, Bertoletti A, Hsu LY, Ong BC (2021): Widely heterogeneous humoral and cellular immunity after mild SARS-CoV-2 infection in a homogeneous population of healthy young men. Emerg. Microbes Infect. 28, 1-26. Epub ahead of print. https://doi.org/10.1080/22 221751.2021.1999777

COVID-19 Treatment Guidelines Panel, Coronavirus Disease 2019 (COVID-19) Treatment Guidelines, National Institutes of Health (2021): Available at https://www. covid19treatmentguidelines.nih.gov/overview/clinical-spectrum/, accessed on October 26, 2021.

De Santis GC, Mendrone A, Langhi D Jr, Covas DT, Fabron A Jr, Cortez AJP, Dinardo CL, Ubiali EMA, Marques JFC Jr, Bordin JO, Rugani MA (2021):Suggested guidelines for convalescent plasma therapy for the treatment of COVID-19. Hematol. Transfus. Cell Ther. 43, 212-213. Epub 2021 Mar 20. https://doi.org/10.1016/i.htct.2021.03.001

Ebinger JE, Fert-Bober J, Printsev I, Wu M, Sun N, Prostko JC, Frias EC, Stewart JL, Van Eyk JE, Braun JG, Cheng S, Sobhani K (2021):Antibody responses to the BNT162b2 mRNA vaccine in individuals previously infected with SARS-CoV-2. Nat. Med. 27, 981-984. Epub 2021 Apr 1. https://doi.org/10.1038/s41591-021-01325-6

Elliott P, Haw D, Wang H, Eales O, Walters CE, Ainslie KEC, Atchison C, Fronterre C, Diggle PJ, Page AJ, Trotter AJ, Prosolek SJ; COVID-19 Genomics UK (COG-UK) Consortium, Ashby D, Donnelly CA, Barclay W, Taylor G, Cooke G, Ward H, Darzi A, Riley S (2021):Exponential growth, high prevalence of SARS-CoV-2, and vaccine effectiveness associated with the Delta variant. Science eabl9551. Epub ahead of print. https://doi.org/10.1126/ science.abl9551

Haas EJ, Angulo FJ, McLaughlin JM, Anis E, Singer SR, Khan F, Brooks N, Smaja M, Mircus G, Pan K, Southern J, Swerdlow DL, Jodar L, Levy Y, Alroy-Preis S (2021): Impact and effectiveness of mRNA BNT162b2 vaccine against SARS-CoV-2 infections and COVID-19 cases, hospitalisations, and deaths following a nationwide vaccination campaign in Israel: an observational study using national surveillance data. Lancet 397 , 1819-1829. Epub 2021 May 5. Erratum in: Lancet 2021 Jul 17; 398(10296): 212. https://doi.org/10.1016/S01406736(21)00947-8

Harvey WT, Carabelli AM, Jackson B, Gupta RK, Thomson EC, Harrison EM, Ludden C, Reeve R, Rambaut A; COVID-19 Genomics UK (COG-UK) Consortium, Peacock SJ,
Robertson DL (2021): SARS-CoV-2 variants, spike mutations and immune escape. Nat. Rev. Microbiol. 19, 409-424. Epub 2021 Jun 1. https://doi.org/10.1038/ s41579-021-00573-0

Hinton DM (2021): The Authorized COVID-19 Convalescent Plasma. USA. Food and Drug Administration (FDA) March 9, 2021. Available at: https://www.fda.gov/media/141477/download.

Khoury DS, Cromer D, Reynaldi A, Schlub TE, Wheatley AK, Juno JA, Subbarao K, Kent SJ, Triccas JA, Davenport MP (2021): Neutralizing antibody levels are highly predictive of immune protection from symptomatic SARS-CoV-2 infection. Nat. Med. 27, 1205-1211. Epub 2021 May 17.https://doi.org/10.1038/s41591-021-01377-8

Kollár R, Bod'ová K (2021): True pandemic state and a lack of capacity of hospitals and mechanical ventilations in Slovakia during the SARS-COV-2 pandemic wave in August 2020-May 2021, Proceedings of the 21st Conference Information Technologies-Applications and Theory, ITAT 2021, 247-254. http://ceur-ws.org/ Vol-2962/paper14.pdf

Levi R, Azzolini E, Pozzi C, Ubaldi L, Lagioia M, Mantovani A, Rescigno M (2021): One dose of SARS-CoV-2 vaccine exponentially increases antibodies in individuals who have recovered from symptomatic COVID-19. J Clin Invest. 2021 June 15; 131(12), e149154. https://doi. org/10.1172/JCI149154

Levin EG, Lustig Y, Cohen C, Fluss R, Indenbaum V, Amit S, Doolman R, Asraf K, Mendelson E, Ziv A, Rubin C, Freedman L, Kreiss Y, Regev-Yochay G (2021): Waning Immune Humoral Response to BNT162b2 Covid-19 Vaccine over 6 Months. N. Engl. J. Med. NEJMoa2114583. Epub ahead of print.https://doi.org/10.1056/NEJMoa2114583

Lopez Bernal J, Andrews N, Gower C, Robertson C, Stowe J, Tessier E, Simmons R, Cottrell S, Roberts R, O'Doherty M, Brown K, Cameron C, Stockton D, McMenamin J, Ramsay M (2021):Effectiveness of the Pfizer-BioNTech and Oxford-AstraZeneca vaccines on covid-19 related symptoms, hospital admissions, and mortality in older adults in England: test negative case-control study. BMJ. 373, n1088. https://doi.org/10.1136/bmj.n1088

Mendrone-Junior A, Dinardo CL, Ferreira SC, Nishya A, Salles NA, de Almeida Neto C, Hamasaki DT, Facincani T, de Oliveira Alves LB, Machado RRG, Araujo DB, Durigon EL, Rocha V, Sabino EC (2021): Correlation between SARS-COV-2 antibody screening by immunoassay and neutralizing antibody testing. Transfusion 61 , 1181-1190. Epub 2021 January 25. https://doi.org/ 10.1111/trf.16268

Narasimhan M, Mahimainathan L, Noh J, Muthukumar A (2021): Silent SARS-CoV-2 Infections, Waning Immunity, Serology Testing, and COVID-19 Vaccination: A Perspective. Front. Immunol.12,730404. https://doi. org/10.3389/fimmu.2021.730404

Patel SY, Carbone J, Jolles S (2019): The Expanding Field of Secondary Antibody Deficiency: Causes, Diagnosis, and Management. Front. Immunol.10,33. https://doi. org/10.3389/fimmu.2019.00033 
Racine-Brzostek SE, Yee JK, Sukhu A, Qiu Y, Rand S, Barone PD, Hao Y, Yang HS, Meng QH, Apple FS, Shi Y, Chadburn A, Golden E, Formenti SC, Cushing MM, Zhao Z (2021): Rapid, robust, and sustainable antibody responses to mRNA COVID-19 vaccine in convalescent COVID-19 individuals. JCI Insight. 2021 October 22;6(20), e151477. https://doi.org/10.1172/jci.insight.151477

Sette A, Crotty S (2021): Adaptive immunity to SARS-CoV-2 and COVID-19. Cell 184, 861-880. Epub 2021 January 12. https://doi.org/10.1016/i.cell.2021.01.007
Wei J, Stoesser N, Matthews PC, Ayoubkhani D, Studley R, Bell I, Bell JI, Newton JN, Farrar J, Diamond I, Rourke E, Howarth A, Marsden BD, Hoosdally S, Jones EY, Stuart DI, Crook DW, Peto TEA, Pouwels KB, Eyre DW, Walker AS; COVID-19 Infection Survey team (2021): Antibody responses to SARS-CoV-2 vaccines in 45,965 adults from the general population of the United Kingdom. Nat Microbiol. 6, 1140-1149. Epub 2021 July 21. https:// doi.org/10.1038/s41564-0 21-00947-3

WHO COVID-19 Dashboard. Geneva: World Health Organization (2020):Available online: https://covid19.who.int/ (Accessed on: November 5, 2021). 